\title{
El ingreso familiar y el acceso de jóvenes a la educación media en Honduras
}

Héctor Díaz Romero*

\section{RESUMEN}

El siguiente documento sustenta empíricamente: la influencia que tiene el ingreso económico familiar en las posibilidades de los jóvenes, para ingresar al sistema de educación media en Honduras, partiendo de la hipótesis siguiente: el ingreso económico familiar influye de manera sistemática la participación de los niños y jóvenes en la educación. Esta investigación utiliza un método econométrico de regresión probabilística para testear la hipótesis, el cual se validó con criterios estadísticos de Akaike y Schwarz para modelos anidados, que resultó en un modelo robusto para explicar de manera bastante aceptable la variable.

Los datos presentados provienen de la Encuesta para el Mejoramiento de Condiciones de Vida (ENCOVI) que aplicó el Instituto Nacional de Estadísticas (INE) en el Año 2004. El acceso a datos más recientes no fue posible, por lo anterior, es probable que alguna de la problemática planteada ya haya sido resuelta por los programas públicos dedicados a la materia.

Este documento demuestra que el ingreso familiar tiene un impacto sistemático sobre el acceso de los jóvenes entre 12 y 18 años al sistema de educación media en Honduras; además, la zona geográfica donde habitan las familias tiene una influencia determinante en tal acceso. Por lo tanto es necesario, establecer políticas públicas encaminadas a solucionar estos problemas.

Palabras claves: Proxy, variable dicotómica, quintil de ingreso, variables de control, STATA SE 10.

\section{ABSTRACT}

The following document has support empirically about: the influence of the family income in the possibilities of young people to enter the middle education system in Honduras, assuming the following hypothesis: the family income systematically

* Universidad Nacional Autónoma de Honduras/ Máster en Políticas Públicas. Universidad de Chile. Facultad de Economía y Negocios. hwdromero@yahoo.es 
affect the participation of children and youth education. This research uses a probabilistic econometric regression to test the hypothesis, which was validated with statistical criteria of Akaike and Schwarz for nested models, which resulted in a robust model to explain quite acceptable variable.

The data presented are from the Encuesta para el Mejoramiento de la Condiciones de Vida (ENCOVI) applied by Instituto Nacional de Estadisticas (INE) in 2004. The most recent data access was not possible and it is likely that some of the issues raised have already been resolved by public programs dedicated to the subject.

This paper shows that family income has a systematic impact on access of young people between 12 and 18 at the middle education system in Honduras, in addition, the geographic area where families living have a crucial influence on such access. Therefore it is necessary to establish public policies aimed at solving these problems.

Key Words: Proxy, two-value, pay quantil, control variable, state SE 10. 


\section{INTRODUCCIÓN}

El sistema de Educación Media en Honduras ayuda a la formación integral de adolescentes, continúa el proceso formativo de la educación primaria, capacita para el ejercicio profesional ${ }^{1}$, y se imparte en dos ciclos; el ciclo común de cultura general, con fines de cultura general, exploración y orientación vocacional; y el ciclo diversificado para continuar la orientación, intensificar los estudios y dar formación profesional en diferentes campos de trabajo ${ }^{2}$. Este objetivo se ha visto mermado por las dificultades que miles de jóvenes hondureños tienen para ingresar la educación media, por factores que se pueden relacionar con antecedentes de tipo económico, cultural, social, entre otros.

De acuerdo con el promedio histórico de la matricula, los jóvenes que participan en el sistema de educación media representan a penas el 24.5\% (entre el año 2002 y el 2008) de todos los alumnos que participan en el sistema de educación de Honduras ${ }^{3}$ (no se incluye el Nivel Preescolar ni universitario).

Este trabajo demuestra mediante un método econométrico que el efecto que tiene el ingreso económico familiar sobre el acceso de los jóvenes entre 12 y 18 años al sistema de educación media en Honduras es cercano a 10 puntos porcentuales, además hace lo mismo con el área de residencia de la familia y con otras variables de interés. Se demuestra que vivir en el área rural disminuye en casi 30 puntos porcentuales la posibilidad de que los jóvenes de 12 a 18 años tengan acceso a la educación media.

Es necesario plantear soluciones de política pública que resuelvan las causas de esta problemática. La educación es vista como productora de capital humano ${ }^{4}$; además, reduce las desigualdades sociales y económicas; por lo tanto, debe plantearse como alternativa para lograr avances significativos en dichas aéreas. Honduras es un país donde el $69 \%$ de su población vive en condiciones de pobreza, lo que hace indispensable invertir de mejor manera los recursos disponibles y la educación es un camino seguro.

\footnotetext{
${ }^{1}$ Articulo 27 de la ley Orgánica de Educación

${ }^{2}$ Articulo 28 de la ley Orgánica de Educación

${ }^{3}$ Secretaria de Educación, estadística educativa 2002-2008

${ }^{4}$ La capacidad productiva del individuo es incrementada por la educación, de acuerdo con la Organización de Estados Iberoamericanos.

${ }^{5}$ CEPAL (2009)
} 


\section{La Investigación}

En un país sumergido en la pobreza y con grandes deseos de aumentar el capital humano de sus habitantes, para disminuir las desigualdades sociales y así mejorar las condiciones de vida que hasta ahora han sido precarias para las mayorías de sus ciudadanos; es necesario preguntarse ¿Es el ingreso económico familiar determinante para el ingreso de los jóvenes hondureños entre 12 y 18 años, que han finalizado la primaria, al sistema de educación media? Esta pregunta se pretende contestar en este trabajo.

\subsection{Importancia del Tema}

Honduras es un país con una fuerte inversión en educación pública, invierte más que el promedio de los países latinoamericanos. En rubros relacionados con la educación, anualmente se gastan en promedio un $24.6 \%{ }^{6}$ (del año 1995 al año 2005) del gasto total del gobierno, que equivale al $7.1 \%$ del PIB. Pero esta mayor inversión no ha resultado en mejores rendimientos, al medir la eficiencia del gasto público en educación, Honduras se encuentra muy por debajo de la media.

Según el Banco Mundial el país posee el promedio más bajo de matricula en el sistema de educación media, comparado con los países de ingreso per cápita equivalentes, por lo tanto en este nivel educativo es necesario priorizar esfuerzos para lograr mejores resultados. Además invertir en educación aumenta el desarrollo humano, reduce la pobreza y consolida la democracia.

\subsection{Análisis Metodológico}

Para evaluar la hipótesis se usará un modelo probabilístico, frente a la evidencia de la existencia de una variable dicotómica. Esta variable considera la posibilidad de entrar o no al sistema de educación media de Honduras, demostrando cómo esta posibilidad se ve influida por el quintil de ingreso al que se pertenece.

Otras variables también se consideran como explicativas, entes estas el sexo, la región geográfica donde se habita, la pertenencia o no a un grupo étnico, el número de personas por hogar y la educación de los padres.

\footnotetext{
${ }^{6}$ Fuente Secretaria de Finanzas Honduras, no se incluye el gasto en educación superior.
} 


\subsection{Planteamientos de éste Trabajo}

Si bien es cierto, las variables que afectan el ingreso y permanencia de jóvenes en edad escolar al sistema de educación media en Honduras son muy variadas, el ingreso familiar se puede considerar como una variable que incide de manera sistemática en este fenómeno. En este trabajo se identifica al menos dos formas en que el bajo ingreso familiar influye en que los jóvenes no ingresen a la educación media; la primera es que un bajo ingreso familiar obliga a los jóvenes a abandonar sus estudios y comenzar a trabajar para contribuir a la economía familiar; la segunda es: no ingresan porque los costos a los que se enfrentan las familias que poseen jóvenes en el sistema de educación media, están sobre las posibilidades que tienen las familias con ingresos del primero y segundo quintil para financiarla.

Lo anterior se demuestra cuando en el año 2004, del total de jóvenes que ingresaron a la educación media, solo el $17.12 \%$ pertenecían al primero y segundo quintil, en cambio el $62.49 \%$ pertenecían al cuarto y quinto quintil (ENCOVI 2004).

\subsection{Estado Actual de la Temática}

El Estado de Honduras ha hecho esfuerzos por mejorar los niveles de educación media del país. Estos esfuerzos se resumen básicamente en la eliminación del ciclo común de cultura general, que pasa a formar parte del ciclo básico. Así que la educación básica consiste en tres ciclos y por lo tanto, se vuelve obligatorio el sistema de educación hasta el noveno grado (tercero de ciclo común en cultura general), el ciclo diversificado se continua tratando como voluntario pero gratuito para todos los jóvenes en edad escolar.

Existen múltiples estudios sobre la educación en Honduras, la mayoría se han concentrado en medir temas de cobertura; eficiencia; calidad; equidad; estándares y evaluación; autonomía y rendición de cuentas; perfeccionamiento docente y financiamiento; por lo que este estudio hace un esfuerzo por medir de manera empírica las causas de la baja matricula en la educación media. Paradójicamente siempre se relaciona la falta de educación como generadora de pobreza, pero pocas veces se relaciona a la pobreza como causante de bajos niveles de educación, y las pocas veces que se menciona no se hace empíricamente sino, como un tema sociológico o histórico; esta observación se hace sin perjudicar la validez científica que estas disciplinas tienen.

Según el informe sobre el progreso educativo en Honduras el principal problema para que los niños y jóvenes en edad escolar no asistan a la escuela, es la situación 
de pobreza en que vive la mayoría de hogares del país ${ }^{7}$. Se plantea entonces la necesidad de implementar ayudas a través de transferencias o de programas compensatorios de los ingresos, como los comedores escolares, los becas o los bonos de útiles escolares.

El segundo aspecto analizado en este informe se refiere a la valoración que los padres tienen por la educación formal de sus hijos, éste es bastante difícil de medir por ser subjetivo, pero existen variables proxi $^{8}$ que pueden dar una tendencia sobre la valoración de los padres por la educación formal.

\subsection{Modelo Econométrico Escogido}

Para la elaboración del trabajo se eligió un modelo probabilístico, debido a que la variable explicada es dicotómica y por tal motivo conviene utilizarlo. Se pretende evaluar el efecto del ingreso en el acceso o no de jóvenes en edades de 12 a 18 años que han terminado la educación primaria al sistema de educación media de Honduras, utilizando otras variables de control que servirán para completar mejor el modelo. Estas variables han mostrado en otros estudios, ser explicativas del modelo.

El modelo que se utilizará $Y_{i}^{*}=\hat{a}_{0}+\hat{a}_{1} \quad \cdot y+\hat{a}_{2} \quad \cdot s+\hat{a}_{3} \quad \cdot a+\hat{a}_{4} \quad \cdot p+\hat{a}_{5} \cdot m+\mu_{i}$ $\operatorname{Pr}\left(Y i=1 \mid \hat{a}_{1} \quad . y, \hat{a}_{2} \quad . s, \hat{a}_{3} \quad . a, \hat{a}_{4} \quad . p, \hat{a}_{5} \cdot m\right)=0 ̈\left(Y i^{*}\right)=O ̈\left(\hat{a}_{1} \quad . y+\hat{a}_{2} \quad . s+\hat{a}_{3} \quad . a+\right.$ $\left.\hat{a}_{4} \quad . p+\hat{a}_{5} \cdot m+\mu_{i}\right)$, si $\mu_{i} N(0,1)$

$Y_{i}=1$ si los jóvenes entre 12 y 18 años ingresaron a algún grado de educación media $Y_{i}=0$ si los jóvenes entre 12 y 18 años no ingresaron a algún grado de educación media

$y$, representa los quintiles de ingreso,

$s$, el sexo,

a, es elárea geográfica,

$p$, es el número de personas que habitan en el hogary

$m$, es la educación de la madre

\footnotetext{
${ }^{7}$ Realizado por la Fundación para la Educación Ricardo Ernesto Maduro Andréu FEREMA en el 2005

${ }^{8}$ Se refiere a variables que por su falta de medición empírica se miden con variables similares o PROXI tales como los sentimientos o los pensamientos referentes a un tema, que se miden con las reacciones físicas a tales sentimientos o pensamientos.
} 


\subsection{Modelo Teórico}

De acuerdo a la evidencia recabada se espera que el acceso de jóvenes al sistema de educación media se vea afectado positivamente a medida que se aumenta el quintil de ingreso. El modelo es relativamente completo, pero deja fuera el segundo aspecto más importante del que habla el Informe Sobre el Progreso de la Educación en Honduras, la importancia que los padres le dan a la educación; se pretende rescatar ese aspecto subjetivo a través de la variable escolaridad de la madre, quien tradicionalmente influye en las decisiones educativas de los hijos.

Así mismo el modelo al ser innovador, puede mejorarse por medio de variables que complementen o expliquen de mejor manera la importante baja que se presenta en el acceso a la educación media en Honduras. La educación es uno de los componentes fundamentales para lograr disminuir la pobreza, alcanzar el desarrollo económico y social, ampliar la democracia y lograr la paz social.

\subsection{Modelo Empírico}

El modelo representa a todos los jóvenes entre 12 y 18 años que pertenecen a un hogar y que han culminado la escuela primaria, de acuerdo con la Encuesta para el Mejoramiento de las Condiciones de Vida ENCOVI 2004, desarrollado con las variables siguientes:

Ingresaron o no a un nivel de educación media: sea este ciclo común de cultura general, tercer ciclo básico o Ciclo Diversificado; esta variable se denomina "media" y toma valor 0 si no ingresó a la media y 1 si ingresó a la media.

El quintil de ingreso al que pertenecen: esta variable se denomina "quintil" y toma valores 1 si pertenece al primer quintil de ingreso, 2 si pertenece al segundo quintil de ingreso, 3 si pertenece al tercer quintil de ingreso, 4 si pertenece al cuarto quintil de ingreso y 5 si pertenece al quinto quintil de ingreso.

El sexo del joven: esta variable se denomina "sexo" y toma valor 1 si es mujer y 2 si es hombre.

Área geográfica de residencia del joven: esta variable se denomina "área" y toma valor 1 si el joven reside en un área urbana y 2 si el joven reside en un área rural.

El grupo étnico al que pertenece el joven: esta variable se denomina "indigena" y toma valor 0 si el joven declara no pertenecer a ninguna grupo racial o indígena 
autóctono y 1 si el joven declara pertenecer a algún grupo racial o indígena autóctono, aunque no conserve su lengua materna.

Escolaridad de la madre: esta variable se denomina "escmadre" y toma valores entre 0 y 13 de acuerdo con los años de escolaridad que ha cursado la madre del joven, 0 significa ningún nivel de escolaridad, contrario a 13 que significa estudios de postgrado.

Número de personas que pertenecen a un hogar: esta variable se denomina "numper" y hace un conteo de todos los miembros de un hogar.

La base de datos se trabajó con el Software estadístico STATA SE 10, para la construcción de las variables se utilizó la Encuesta para el Mejoramiento de las Condiciones de Vida de los Hogares ENCOVI 2004, practicada en Honduras, de la cual se unió los datos referentes a personas y a viviendas, utilizando para ello el del identificador del hogar.

Luego se rescató únicamente los datos que se relacionan con un hogar, por lo que los resultados sólo son aplicables a hogares. Tomando los datos por hogar de los jóvenes de 12 a 18 años que habían finalizado la educación primaria, se identificaron los que se matricularon y los que no se matricularon en un colegio de educación media o en el tercer ciclo en un centro de educación básica de Honduras.

\section{Metodología}

\subsection{Test para la selección del modelo}

Se probaron tres diferentes modelos para encontrar el mejor, bajo el criterio Akaike ( $\mathrm{ACl}$ ) y Schwarz $(\mathrm{BIC})$ que se utilizan para modelos anidados, reforzado por el nivel de significancia global del modelo o R2 dando como resultado que el modelo 1 (ver cuadro 1) se identificó como el mejor.

Las pruebas se realizaron bajo el siguiente procedimiento y criterio: la principal variable que se testea es ingreso familiar, por lo que se probó tres alternativas posibles, quintil de ingreso del hogar "quintil", ingreso total del hogar "ytothg" e ingreso del hogar percápita "yperhy" resultando con menor BIC y AIC el modelo que incluía el quintil de ingreso del hogar, por lo tanto se optó por este modelo ya que estadísticamente explica mejor la influencia del ingreso familiar en el acceso de los jóvenes a le educación media en Honduras. 


\begin{tabular}{|c|c|c|c|}
\hline \multicolumn{4}{|c|}{ Cuadro 1} \\
\hline Variable & Modelo 1 & Modelo 2 & Modelo 3 \\
\hline quintil & 0.2710 & & \\
\hline valor $\mathrm{P}$ & 0.0000 & & \\
\hline yperhg & & 0.0001 & \\
\hline valor $P$ & & 0.0000 & \\
\hline ytothg & & & 0.0000 \\
\hline valor $\mathrm{P}$ & & & 0.0000 \\
\hline sexo & 0.0979 & 0.0802 & 0.0836 \\
\hline valor $P$ & 0.0410 & 0.0870 & 0.075 \\
\hline area & -0.7500 & -0.9510 & -0.963 \\
\hline valor $P$ & 0.0000 & 0.0000 & 0.0000 \\
\hline nper & -0.0300 & -0.0290 & -0.0446 \\
\hline valor $P$ & 0.0390 & 0.0410 & 0.002 \\
\hline indigena & 0.0276 & 0.0675 & 0.0766 \\
\hline valor $P$ & 0.8720 & 0.6870 & 0.6480 \\
\hline escmadre & 0.0208 & 0.0293 & 0.0295 \\
\hline valor $\mathrm{P}$ & 0.0040 & 0.0000 & 0.0000 \\
\hline escpadre & 0.0052 & 0.0100 & 0.0102 \\
\hline valor $\mathrm{P}$ & 0.5250 & 0.2090 & 0.2010 \\
\hline cons & 0.2640 & 1.2800 & 1.3600 \\
\hline valor $P$ & 0.0680 & 0.0000 & 0.0000 \\
\hline Seudo R2 & 0.1581 & 0.1258 & 0.1268 \\
\hline AIC & 3730 & 3917 & 3922 \\
\hline $\mathrm{BIC}$ & 3768 & 3966 & 3971 \\
\hline
\end{tabular}

El segundo test que se realizó fue el de significancia de las variables, en este caso también se probaron tres modelos diferentes. El Cuadro 2 muestra estos modelos.

\begin{tabular}{|c|c|c|c|}
\hline \multicolumn{5}{|c|}{ Cuadro 2 } \\
\hline Variable & Modelo 1 & Modelo 2 & Modelo 3 \\
\hline quintil & 0.271 & 0.272 & 0.272 \\
\hline valor P & 0.0000 & 0 & 0 \\
\hline sexo & 0.0979 & 0.0975 & 0.0938 \\
\hline valor P & 0.041 & 0.0420 & 0.05 \\
\hline area & -0.75 & -0.752 & -0.0754 \\
\hline valor P & 0.0000 & 0.0000 & 0.0000 \\
\hline nper & -0.03 & -0.0299 & -0.0303 \\
\hline valor P & 0.039 & 0.0390 & 0.037 \\
\hline indigena & 0.0276 & 0.0250 & \\
\hline valor P & 0.872 & 0.8840 & 0.0215 \\
\hline escmadre & 0.0208 & 0.0215 & 0.0030 \\
\hline valor P & 0.004 & 0.0030 & \\
\hline escpadre & 0.516 & & 0.2800 \\
\hline valor P & 0.525 & 0.2710 & 0.0520 \\
\hline cons & 0.264 & 0.0600 & 3737 \\
\hline valor P & 0.068 & 3728 & 3773 \\
\hline AIC & 3730 & 3771 & \\
\hline BIC & 3778 & & \\
\hline
\end{tabular}


Las variables de control se eligieron tomando en cuenta otros estudios, pero al igual que la variable ingreso, se eliminaron las que tenían valor P no significativo; es decir, que a un $95 \%$ de confianza se descartaban como explicativas del modelo. Estas son: escolaridad del padre "escpadre" y grupo étnico "indigena".

Al utilizar siempre los criterios BIC y AIC resultó que el modelo sí mejoraba quitándole la variable "escpadre"; en cambio, al quitarle la variable "indigena" a pesar de no ser estadísticamente significativa, éste empeoraba, por lo que se decidió incluirla.

\subsection{Testeando la hipótesis}

Se ha construido la hipótesis preguntándose ¿El ingreso familiar afecta el acceso de jóvenes entre 12 y 18 años al sistema de educación media de Honduras?

El Gráfico 1 muestra cómo se comporta la variable explicada si sólo se compara con el quintil de ingreso

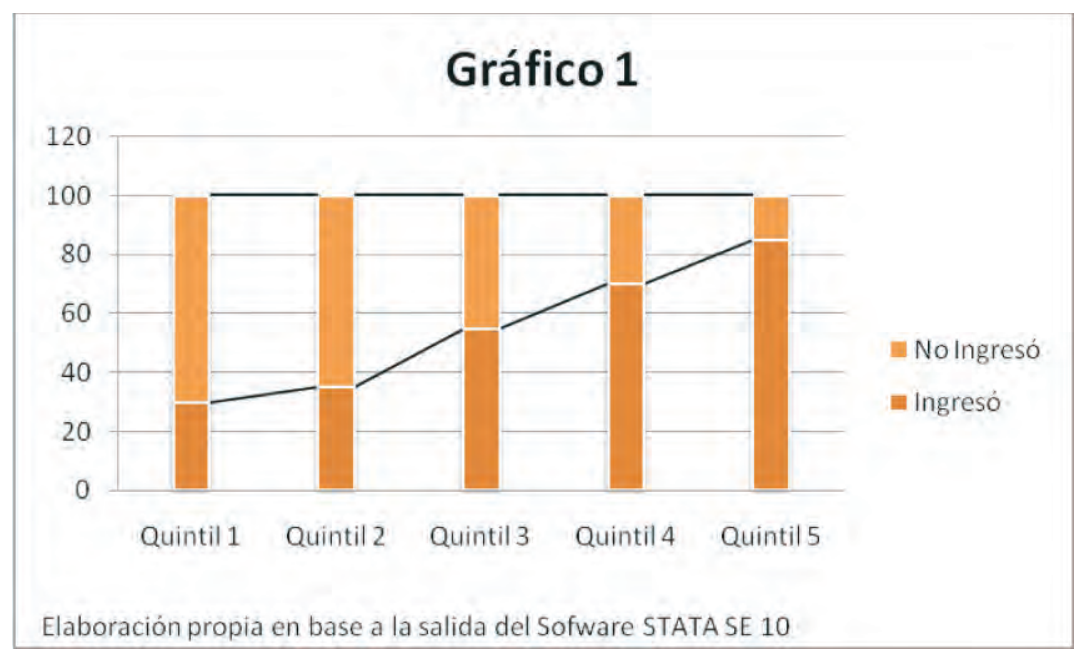

Gráficamente se observa que la proporción de estudiantes que ingresaron a la media aumenta a medida que se avanza en lo quintiles de ingreso, los dos quintiles más pobres tienen una proporción de participación menor que el ultimo quintil solo, por lo tanto, la hipótesis planteada de que el ingreso afecta de manera sistemática el acceso de los jóvenes a la educación media en Honduras, es reforzada con la información empírica de la ENCOVI 2004. 


\section{Resultados del Modelo}

\subsection{Resultados del Modelo Empírico}

El modelo es estadísticamente aceptable ya que posee un seudo R2 de .1581 (ver cuadro 1), es decir que las variables utilizadas explican el acceso de jóvenes entre 12 y 18 años al sistema de educación media en Honduras en algo más del 15\%, el resto es explicado por otras variables que no se encuentran el modelo ya sea porque no son empíricamente medibles o porque se omitieron.

\section{Cuadro 3}

\section{$\mathrm{y}=\operatorname{Pr}($ media $) \quad$ (predict) \\ pseudo R2 $=.60022848$}

\begin{tabular}{|c|c|c|c|c|c|c|c|}
\hline Variable & $\mathbf{d y} / \mathbf{d x}$ & Err. Est & $\mathbf{z}$ & $\mathbf{P}>\mathbf{z} \mid$ & \multicolumn{2}{|c|}{ [ I. C. 95\% ] } & $\mathbf{x}$ \\
\hline quintil & 0.1049118 & 0.00745 & 14.09 & 0.000 & 0.09032 & 0.11951 & 3.26104 \\
\hline sexo & 0.0376702 & 0.0185 & 2.04 & 0.042 & 0.00144 & 0.0739 & 1.52945 \\
\hline area & -0.2903859 & 0.0212 & -13.7 & 0.000 & -0.331936 & -0.2488 & 1.30429 \\
\hline nper & -0.0115512 & 0.0056 & -2.05 & 0.04 & -0.0226 & -0.0005 & 3.98988 \\
\hline indigena* & 0.009635 & 0.0618 & 0.1600 & 0.876 & -0.111489 & 0.13076 & 0.020245 \\
\hline escmadre & 0.0083238 & 0.0027 & 3.0600 & 0.002 & 0.003 & 0.01365 & 2.21595 \\
\hline
\end{tabular}

* dy/dx es el cambio marginal para una variable dummy de 0 a 1 Elaboración propia de la salida del software STATA SE 10

El ingreso familiar es la principal variable que se pretendía testear por considerarse significativa, se demostró que efectivamente influye de manera sistemática en el acceso de un joven de 12 a 18 años que ha finalizado la primaria, al sistema de educación media de Honduras; aunque sorpresivamente afecta marginalmente más vivir en una zona rural, que el nivel de ingreso familiar (ver cuadro 3 ) esto puede ser explicado por la menor proporción de establecimientos de educación media que hay en la zona rural. También se podría plantear como multi causal, ya que en la zona rural se concentra más el índice de pobreza y la aplicabilidad que tiene la educación con el trabajo es más difícil de asociar; por lo tanto, la valoración por la educación formal tiende a ser menor.

Con respecto a la variable de: "ser o no indígena" resulta claramente no significativa, pero la desmejora del modelo que se observó en el test de significancia de las variables, demuestra que hay efectos que el modelo no captura en esta variable y por lo tanto no se muestran estadísticamente. Pertenecer a grupos familiares más grandes, afecta negativamente el acceso de jóvenes al ingreso a la media, lo cual también era esperado ya que en familias más numerosas existe competencia por los 
recursos, disminuyendo la probabilidad de que la familia financie la educación. Con la variable "escolaridad de la madre" se pretende rescatar en parte la valoración que tienen los padres por la educación, el resultado fue efectivamente una mayor educación de la madre aumenta la probabilidad de ingresar a la media a un joven de entre 12 y 18 años.

Un dato que se lamenta corroborar es que ser hombre aumenta la probabilidad de ingresar a la media, esto es inaceptable en un país donde la mayoría de sus habitantes son mujeres y donde estas deberían constituir la mayor fuerza de trabajo yser un recurso con alto nivel de capital humano.

\subsection{Validación del Modelo Empírico}

Al realizar el test de correlación se estableció como limite 0.8 que es el que normalmente se utiliza para este tipo de modelos econométricos. Al evaluar las variables se observó que si bien algunas tienen correlación, tal es el caso de las variables "escolaridad de la madre" con la variable "sexo" que tienen una correlación positiva de 0.23 , "escolaridad de la madre" con el "quintil de ingreso" con correlación positiva de 0.20 y "escolaridad de la madre" con el número de personas en el hogar" con una correlación negativa de 0.19.

Esta correlación no es fuerte en ninguno de los casos es cercano al limite establecido, por lo que no existe multicolinealidad en las variables.

La estimación se establece bastante acertada ya que por definición este modelo es Heterocedastico, las variables no están correlacionadas entre si, así que el modelo se adapta de manera bastante satisfactoria para responder la interrogante planteada.

\section{Implicancias de política}

La evidencia empírica ha dejado en manifiestos dos principales problemas para ser atendidos. El primero es que el ingreso familiar afecta el acceso de los jóvenes entre 12 y 18 años al sistema de educación media en el país; por lo tanto, cualquier programa que tenga como componente principal el complemento de los ingresos económicos de las familias con jóvenes en este rango etéreo, logrará aumentar el acceso de los jóvenes al sistema de educación media.

El segundo es que la ruralidad al ser la variable de mayor incidencia negativa, ya sea por concentración de pobreza, por densidad de centros o por valoración de la educación, es el punto focal donde se deben dirigir los programas de inserción 
educativa media en el país. El aporte de este documento y los sucesivos en la materia deben poner especial interés en estas variables y crear mecanismos para medir el impacto que tengan los programas públicos de inserción al sistema educativo en ellas.

Finalmente, si bien la evidencia empírica no pudo demostrar la desventaja que tienen los grupos poblacionales pertenecientes a minorías indígenas, sí se considera necesario crear mecanismos de monitoreo de estos grupos poblacionales, ya que usualmente se ven más afectados por problemáticas sociales y económicas, como la pobreza y la exclusión social, siendo este caso muy similar y que requiere del diseño de soluciones públicas encaminadas a su mejoramiento.

\section{CONCLUSIONES}

\subsection{Conclusiones a la investigación}

La temática planteada en esta investigación es de suma importancia para el país, ya que el mejoramiento educativo es un paso necesario para alcanzar el desarrollo económico y social. Indagar en las causas del bajo nivel educativo promedio del país, es vital para buscar soluciones más eficientes para esta problemática.

La concentración en el grupo de edad de 12 a 18 que se plantea en esta investigación, es un esfuerzo por caracterizar de mejor manera a un grupo poblacional que en su mayoría carece de alternativas a sus demandas personales; por lo tanto, incide sobre otros fenómenos sociales como la desocupación, que consecuentemente derivará en aumentos en los índices de violencia e inseguridad.

Esta investigación también emite una alerta sobre las desigualdades sociales, ya que, según muestra la evidencia, con la actual estructura de acceso a la educación media, se asentaran más las desigualdades sociales. En la medida que las personas de los quintiles más pobres tengan menor probabilidad de continuar educándose, se afectarán las proyecciones de desarrollo del país y se imposibilitara el alcanzar la paz social.

\subsection{Conclusiones al Modelo Empírico}

Efectivamente, con los datos que se poseen se puede decir que el nivel de ingreso económico familiar afecta el acceso de jóvenes entre los 12 y 18 años, que han finalizado la educación primaria, al sistema de educación media de Honduras, esta variable explica en $10.49 \%$ dicha probabilidad de acceso, en un modelo que explica 
cerca del 15\% de la problemática planteada. De igual manera se observa que vivir en un área rural afecta de manera negativa en casi 30\% el tener acceso a la educación media en Honduras, esta es la variable más significativa del modelo y por lo tanto se recomienda sea tomada en cuenta en futuras investigaciones.

Dado el contexto Nacional en donde la mayoría de la población son mujeres, el dato que resulta preocupante es que ser hombre aumenta la posibilidad de ingresar a la media, esto requiere de una política de género que se encargue de equipara las posibilidades de las mujeres y los hombres a ingresar al sistema de educación media en Honduras.

\section{SUGERENCIAS DE INVESTIGACIÓN}

Ya que se demostró empíricamente una relación entre el ingreso económico familiar y el acceso de jóvenes a la educación media, es importante para temas de política pública conocer cual es el monto aproximado con el cual se deben complementar los ingresos, al menos para que el $50 \%$ de los jóvenes del primero y segundo quintil puedan ingresar a la educación media; Además es importante realizar estudios sobre las variables no explicadas en este estudio como la valoración por la educación y de los antecedentes culturales de las familias tales como el machismo, la religión o la posición familiar sobre al embarazo adolecente.

\section{AGRADECIMIENTOS}

Se agradece a Bolívar Ordoñez, Profesor titular de la Carrera de Administración Pública de laUNAH, por sus comentarios de este trabajo.

\section{BIBLIOGRAFÍA}

Fundación para la Educación Ricardo Ernesto Maduro Andreu (2005) "Informe sobre el progreso educativo de Honduras"Tegucigalpa. Honduras.

Congreso Nacional de Honduras (1996) "Ley Orgánica de Educación Honduras Decreto 76/1966" Tegucigalpa Honduras.

\section{Información Web}

Sistemas Educativos Nacionales, Honduras. Organización de Estados Iberoamericanos. Web:http://www.oei.es/quipu/honduras/

Encuesta para el Mejoramiento de las Condiciones de Vida 2004 
Instituto Nacional de Estadísticas Honduras

Web: http://www.ine-hn.org/sociales/encuestas/ine/encovi/encovi04/survey0/ index.html

Sistema de Estadística Educativa. Secretaria de Educación Honduras Web:

http://www.se.gob.hn/index.php?a=Webpage\&url=ESTADISTICAS_educativas 